\title{
La descodificación biológica y las alteraciones craneofaciales, una mirada desde las ciencias básicas
}

\section{Biological decoding and craniofacial alterations, a view from basic sciences}

\author{
Nayely Chilanguad ${ }^{1}$ (D) Nicole Salazar Ríos ${ }^{1}$ (D) $\mid$ Ximena Zúñiga Daza ${ }^{1}$ (D) Martha Lucía Rodriguez $^{2}$ (iD \\ | Carlos Humberto Valencia Llano ${ }^{3}$
}

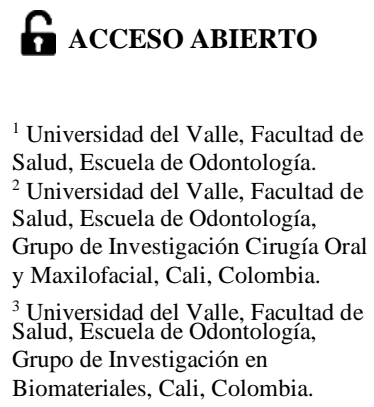

Citación

Chilanguad N, Salazar Ríos N, Zúñiga Daza X, Rodriguez ML, Valencia Llano CH. La descodificación biológica y las alteraciones craneofaciales, una mirada desde las ciencias básicas. Rev Estomatol. 2020;28(2):17-22. DOI: $10.25100 /$ re.v28i1.10829

\section{Correspondencia}

Miguel Angel Casillas Santana Address: Avenida Manuel Espinoza Yglesias 31 Poniente 1304, Los Volcanes, 72410, Puebla, Puebla, Mexico. Phone: 5244 48467645

E-Mail: miguel.casillas@ correo.buap.mx

\section{Copyright:}

(C) Universidad del Valle.

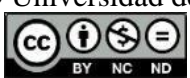

\begin{abstract}
RESUMEN
Antecedentes: La Descodificación Biológica propone la correspondencia entre el estrés ante una situación desbordante y la aparición de síntomas. La ciencia ha logrado profundizar en los componentes biológicos de los trastornos del desarrollo que podrían explicar lo que ocurre en algunos preceptos de esta teoría.

Objetivo: Determinar a partir de la revisión de literatura científica, la relación entre el estrés y algunas hormonas, con las alteraciones craneofaciales.

Materiales y métodos: Se realizó una búsqueda en SciELO, PubMed y SCOPUS buscando artículos relacionados con el estrés, trastornos fisiológicos y esqueléticos y la hormona de crecimiento

Resultados: Se encontraron 17 artículos que relacionan el estrés con alteraciones fisiológicas, 18 artículos que muestran del el papel de los el estrés en el sistema nervioso central y la alteración de la hormona de crecimiento, además 16 artículos que relacionan la hormona de crecimiento con alteraciones esqueléticas craneofaciales Conclusión: se encontró evidencia que muestran cómo algunos supuestos de la teoría de la "Descodificación biológica" pueden ser explicados a partir del papel del estrés y de los estresores que podrían estimular respuestas a nivel del sistema nervioso central y llevar a cambios en estructuras óseas en pacientes en periodo de crecimiento o maduración ósea.
\end{abstract}

\section{PALABRAS CLAVE}

Descodificación biológica, Descodificación Genética, Estrés, Estresores, Alteraciones craneofaciales, hormona de crecimiento.

\section{ABSTRACT}

Background: Biological Decoding proposes the correspondence between stress in an overwhelming situation and the appearance of symptoms. Science has managed to investigate into the biological components of developmental disorders that could explain what happens in some precepts of this theory.

Objective: To determine from the review of scientific literature, the relationship between stress and some hormones, with craniofacial alterations.

Materials and methods: A search was carried out in SciELO, PubMed and SCOPUS looking for articles related to stress, physiological and skeletal disorders and growth hormone

Results: 17 articles were found that relate stress with physiological alterations, 18 articles that show the role of stress in the central nervous system and alteration of growth hormone, in addition 16 articles that relate growth hormone with alterations craniofacial skeletal

Conclusion: evidence was found that shows how some assumptions of the "biological decoding" theory can be explained from the role of stress and stressors that could stimulate responses at the level of the central nervous system and lead to changes in bone structures in patients in a period of bone growth or maturation.

\section{KEY WORDS}

Biological decoding, Genetic decoding, Stress, Stressors, Craniofacial disorders, growth hormone. 


\section{Relevancia Clínica}

La descodificación biológica en la atención clínica es un medio complementario y no invasive que permite obtener un diagnóstico más preciso mediante una valoración integral tanto del saber como del ser del individuo, al escuchar como el cuerpo procesa y expresa determinantes biopsicosociales desencadenantes de la enfermedad. Estos factores tanto ambientales como hormonales, físicos y emocionales, pueden relacionarse con el estrés y dar paso a una condición patológica. Por todo lo anerior, la descodificación juega un papel importante en el análisis de las alteraciones odontológicas dado que en estos casos se deben considerer tanto la valoración física como la emocional y social.

\section{Introducción}

La Descodificación Biológica (DB) es una teoría que propone la existencia de una correspondencia entre el estrés que puede presentar un individuo al exponerse a una situación determinada y la aparición de un síntoma, el desarrollo de alteraciones o el comienzo de enfermedad; en otras palabras, se plantea cómo, una persona que durante su vida ha tenido alguna situación conflictiva que lo haya marcado estará propenso a la aparición de algunas enfermedades, en un concepto también conocido como "Bioneuroemoción". 1

La DB se originó a partir de 5 leyes biológicas: la primera se llama Conflicto biológico que es cuando la persona entra en shock por una experiencia vivida y que ha generado algún tipo de sentimiento corporal. En la segunda se dan manifestaciones de estrés, preocupación, insomnio entre otras. La tercera es la aceptación de que todos los seres humanos tenemos conflictos. La cuarta es encontrar la solución a la causa y la última refiere a cuando el síntoma manifestado en el individuo logra ser superado y sobrevive a dicha causa. ${ }^{2}$

Los conflictos pueden ser solucionados o no por el cerebro; cuando esto no se resuelve, se envía una señal al cuerpo para que reaccione ante tal problema, y es el cerebro en la mayoría de los casos el que genera la solución para poder continuar con nuestro ciclo de vida, encontrando el equilibrio. El equilibrio es una armonía física, emocional y espiritual. ${ }^{3}$

El componente físico es la zona donde se expresa el problema, es decir donde se refieren los signos o síntomas, por lo que la persona asiste a una consulta, el punto por el cual se debe partir. La parte emocional o mental son nuestros pensamientos y lo que conlleva al conflicto y por ende a los síntomas; lo espiritual refleja donde se ha generado principalmente el problema, resentimiento o cualquier sentimiento. ${ }^{2,3}$
Las investigaciones sobre el estrés y su influencia en la condición de salud no es nueva. En el siglo XIX se describió que los organismos son capaces de autorregularse para mantenerse estables ante las presiones del medio externo, posteriormente se diferencian los conceptos "Eustrés" como la respuesta que permite responder ante una situación adaptándose a ella para sobrevivir y el "Distrés" caracterizado por un contenido emocional alto propiciado por experiencias nocivas que pueden originar efectos adversos en la salud del individuo. ${ }^{4}$

El estrés es definido “... como un estímulo puntual, agresivo o no, percibido como amenazante para la homeostasis". 5 En las alteraciones relacionadas con el estrés, se da inicialmente una respuesta adaptativa que involucra varios núcleos cerebrales y en las situaciones de estrés crónico se producen respuestas aún más complejas que pueden convertirse en factores de riesgo para varias enfermedades. . $^{5}$

El objetivo de esta revisión fue determinar a partir de la consulta de textos de literatura científica, la relación entre el estrés y algunas hormonas con las alteraciones craneofaciales.

\section{Materials and methods}

\section{Criterio de búsqueda}

La búsqueda en Google de los términos "Descodificación biológica" y "Biological decoding" arrojó más de 5.000 resultados para la primera expresión y más de 50.000 para la segunda, lo que es un indicativo de la acogida de esta teoría en las redes sociales; sin embargo no se encuentran referencias al buscar estos términos en SciELO, PubMed y SCOPUS.

\section{Criterios de eligibilidad}

Una lectura simple de los conceptos de la DB expuestos en los párrafos anteriores lleva a plantear al estrés como el activador de todo el proceso: el paciente sufre la experiencia traumática, por algunas circunstancias no logra manejarlo bien y en consecuencia se disparan los síntomas de la enfermedad, lo cual se describe de forma similar en la literatura científica, donde el estresor origina inicialmente una reacción adaptativa pero también puede llevar a efectos deletéreos. $^{4}$

\section{Revisión de Literatura}

Con el fin de comprender el mecanismo como el estrés puede ocasionar la aparición de trastornos craneofaciales se realizó inicialmente una búsqueda en las bases de datos 
SciELO, PubMed y SCOPUS sobre estrés y alteraciones sistémicas, para posteriormente buscar relaciones entre el estrés y las alteraciones craneofaciales.

Son abundantes las investigaciones que muestran la relación entre el estrés y diversas condiciones patológicas; por ejemplo en el caso del cáncer se reconoce la relación entre el estrés, el desarrollo del cáncer y la respuesta al tratamiento; ${ }^{7-10}$ también se ha planteado su influencia en trastornos reproductivos en mujeres ${ }^{11}$ y con el síndrome metabólico. ${ }^{12}$

El estrés es un aspecto inherente a la condición humana, que se manifiesta en diversos grados y diferentes efectos en las personas. ${ }^{13}$ Cada vez que una persona se encuentre en una situación de estrés, este va a ser percibido por el sistema nervioso central (SNC), ${ }^{5}$ generando una respuesta biológica, estimulando el hipotálamo y activando el eje Hipotálamo-pituitario-adrenal, ${ }^{14,15}$ liberando neuropéptidos y hormonas del estrés como catecolaminas; noradrenalina, adrenalina, glucocorticoides y hormona de crecimiento (GH), entre otras, ${ }^{16-18}$ de esta forma estos actúan sobre los sistemas efectores de la periferia del cuerpo como por ejemplo el sistema inmunológico, cardiovascular, muscular, óseo y demás tejidos, desencadenando una serie de acontecimientos y alteraciones en el cuerpo humano. ${ }^{7,8,19-21}$

En el estrés crónico se desencadena la liberación de glucocorticoides los cuales desregulan el sistema, ${ }^{22,23}$ ayudando en procesos de inflamación y contribuyendo al riesgo de desarrollar enfermedades. ${ }^{16,21}$

Los factores de transcripción que responden a glucocorticoides son reguladores primarios de la inflamación que se activan durante la señalización de la hormona del estrés; ${ }^{24}$ los glucocorticoides actúan directamente sobre las células óseas, lo que provoca una disminución de la viabilidad de los osteocitos, disminución de la función de los osteoblastos (células productoras de hueso) debido a la reducción del factor de crecimiento insulínico (IGF-2) y una prolongación de la viabilidad de los osteoclastos. Por tanto, el estrés puede afectar negativamente a la salud ósea mediante la modulación de los glucocorticoides endógenos, dando como resultado una disminución en la formación y crecimiento óseo. ${ }^{8,25}$

La hormona del crecimiento $(\mathrm{GH})$ es secretada por la hipófisis anterior, ${ }^{26,27}$ tiene una secreción pulsátil con concentraciones dependientes de la edad que se caracteriza por una secreción baja en el período prepuberal, un aumento en la pubertad y una disminución en la vejez ${ }^{28}$ y junto con el factor de crecimiento similar a la insulina I (IGF-I), juegan un papel importante en la estimulación del crecimiento y el metabolismo óseo, especialmente en lo que respecta al aumento de la masa ósea, actuando principalmente en aquellas partes craneofaciales donde tiene lugar la osificación epifisaria y en las áreas que se adaptan a este crecimiento. ${ }^{5,29}$

Se ha reportado que la GH estimula la aposición ósea a través de la acción de los osteoblastos que agregan tejido mineralizado en la superficie ósea externa (aposición periostica), e indirectamente por medio de las fuerzas musculares que actúan sobre los huesos, siendo regulada por los efectos anabólicos de la GH y el IGF-1 sobre el tejido muscular. ${ }^{21,30}$

Las variaciones en los niveles de la $\mathrm{GH}$ pueden producir cambios en la morfología ósea y llevar a que, por ejemplo, se pueda presentar un atraso en el crecimiento de las estructuras faciales, dando lugar a una cara morfológicamente alterada. La mayoría de los estudios informan retroposición mandibular, ángulo gonion amplio y aumento relativamente menor de la altura facial, el maxilar parece estar afectado en menor medida que la mandíbula, aunque algunos estudios muestran su retroposición. ${ }^{31}$

Los niños con deficiencia de hormona del crecimiento (GHD), se describen en la literatura como poseedores de un cráneo grande y un rostro infantil donde su inteligencia no se ve afectada, permaneciendo dentro de los límites normales para su edad, estos niños son más bajos que sus compañeros y poseen una capa más gruesa de tejido adiposo subcutáneo. ${ }^{32}$

Los estudios cefalométricos demostraron una reducción en las dimensiones de las bases craneales anterior y posterior y la mandíbula más pequeña. ${ }^{33}$ Además, se evidenció que las niñas con GHD tienen mandíbulas significativamente más pequeñas, es decir que su maxilar y mandíbula están retrognáticas, mientras que los niños se caracterizan por la base craneal plana, ambos maxilares retro posicionados, mandíbula retro inclinada y la base craneal subdesarrollada. ${ }^{34,35}$

Los niños y adolescentes con baja estatura causada por la GHD, ${ }^{36}$ pueden enfrentar dificultades en la vida cotidiana causadas por limitaciones físicas y sociales relacionadas con la altura como por ejemplo, dificultades para alcanzar objetos en lugares altos, postura inadecuada, estigmatización, el acoso, baja autoestima, variación de la imagen corporal, falta de competencias sociales esperadas por la edad y aislamiento social, entre otras. ${ }^{37-39}$

Se ha reportado que una de las terapias para estas alteraciones es la aplicación de $\mathrm{GH}$, introducida por primera vez en 1985, está aprobada por la Agencia de Alimentos de EEUU y la Administración de Drogas (FDA) para acelerar el crecimiento y aumentar la estatura adulta en pacientes con GHD. ${ }^{40}$ Esta terapia acelera el crecimiento 
cartilaginoso, generando que la tasa de crecimiento de los huesos se desarrolle a través de la osificación intramembranosa, incluidas las suturas. ${ }^{41}$

Además, el efecto de la terapia en altura final depende del tiempo que sea empleada la hormona, la dosis, su frecuencia de administración y edad de inicio del tratamiento. ${ }^{32}$

Los niños que reciben terapia de reemplazo de hormona del crecimiento a largo plazo (durante dos años) experimentan un mayor crecimiento craneofacial, particularmente con respecto a la altura de la rama mandibular. ${ }^{42}$

Por otro lado, Rongen y col. examinaron a niños con síndrome de Turner el cual se define por la pérdida total o parcial de un cromosoma $X$ y se diagnostica principalmente en la infancia o adolescencia; este síndrome evidencia una deficiencia en el crecimiento corporal y desarrollo en las estructuras craneofaciales. $^{43,44}$ Los niños tratados con $\mathrm{GH}$ demostraron un crecimiento estadísticamente significativo de las ramas mandibulares en comparación con un grupo de control transversal. El crecimiento se asoció con la altura de las ramas mandibulares, no con la longitud de la mandíbula, maxilar, o base craneal anterior. $^{23}$

Los estudios de Juloski et al. demostraron que la GH también ejerce un efecto positivo sobre el crecimiento maxilar. Sin embargo, el aparente aumento de la longitud del maxilar no da como resultado un posicionamiento adecuado (protrusión) de éste. 45,46 Aunque fue evidente un aumento en las dimensiones lineales, no se observaron cambios estadísticamente significativos en las medidas angulares o la relación de estatura facial. ${ }^{32}$

El efecto de la administración de $\mathrm{GH}$ se observa con respecto a la altura facial posterior y la rama mandibular. Sin embargo, esta hormona no puede compensar la ausencia del cromosoma $\mathrm{X}$ y equilibrar las características craneofaciales. También se debe enfatizar que la posición sagital y vertical del maxilar mejoró ligeramente, lo que podría sugerir un efecto positivo de la $\mathrm{GH}$ en su posición. ${ }^{32}$

La terapia puede corregir parcialmente el perfil facial y la oclusión de los dientes. ${ }^{47}$ Sin embargo, también existe evidencia que el tratamiento a largo plazo con GH podría producir efectos secundarios como por ejemplo, conducir al desarrollo de rasgos característicos de la acromegalia, como el aumento de las dimensiones de los pies, las manos o la mandíbula. ${ }^{48}$ También puede tener un efecto negativo en el perfil facial y tratamiento de ortodoncia en caso de asimetría facial o prognatismo mandibular, todos los estudios demuestran consistentemente que la longitud mandibular depende principalmente del crecimiento de sus ramas. ${ }^{32}$

Algunos autores sugieren la necesidad de prestar especial atención durante la terapia con $\mathrm{GH}$ a largo plazo, ya que existe la preocupación de que podría dar lugar a un crecimiento excesivo de la mandíbula y una maloclusión prognática. ${ }^{45,47}$

\section{Resultados}

Al utilizar criterios de búsqueda como estrés y alteraciones fisiológicas en las bases de datos consultadas se encontraron 17 artículos científicos que aportan a la compresión del papel que juegan los estresores en los trastornos fisiológicos al iniciar el proceso a nivel del sistema nervioso central; se encontraron reportes de influencia en temas tan diversos como: cáncer, enfermedad autoinmune, anomalias en piel, trastornos hormonales, trastornos periodontales, etc.

Cuando se revisó acerca de las relaciones entre el estrés y las alteraciones en tejido óseo se encontraron 18 artículos científicos que resaltan el papel de la hormona de crecimiento, los temas de los trabajos fueron principalmente osteoporosis, talla corta y síndrome de Turner.

Al revisar utilizando términos de búsqueda como hormona de crecimiento y alteraciones craneofaciales, se hallaron 16 artículos con temas como desarrollo craneofacial, erupción dental, y madurez dental.

\section{Discusión}

La Descodificación Genética (DG) es una teoría que propone la existencia de una correspondencia entre el estrés que puede presentar una persona al exponerse a una situación determinada y la aparición de un síntoma, el desarrollo de alteraciones o el comienzo de enfermedad; en otras palabras, se plantea cómo una persona que durante su vida ha tenido alguna situación conflictiva que lo haya marcado, estará propenso a la aparición de algunas enfermedades. ${ }^{1-3}$

Los seguidores de la DG han propuesto que una situación estresante que sufra un niño o joven en edad de desarrollo podría ocasionar un trastorno esquelético en sus maxilares, alteraciones en las relaciones de la articulación temporo mandibular (ATM) o alteraciones en la erupción y/o maduración dental. 
En esta revisión bibliográfica se buscó correlacionar al estrés, hormona de crecimiento y alteraciones dentofaciales como una evidencia que pueda soportar la teoría de la DG. El análisis de resultados mostró una relación entre el estrés originado en situaciones conflictivas y las alteraciones de algunas hormonas como corticoides y $\mathrm{GH}$ que podrían explicar los cambios en las estructuras óseas y en las discrepancias maxilofaciales que se plantean en la teoría de la descodificación biológica.

\section{Conclusión}

En la literatura científica hay suficiente evidencia que muestran cómo algunos de los supuestos de la teoría de la "Descodificación biológica" pueden ser explicados a partir del papel del estrés y de los estresores como "disparadores" de síntomas que podrían estimular respuestas a nivel del sistema nervioso central y llevar a cambios en estructuras óseas en pacientes en periodo de crecimiento o maduración ósea, lo que sugiere la necesidad de valorar al paciente mirando más allá de los síntomas con el fin de realizar un verdadero manejo integral de su problema de salud.

\section{Declaración de conflicto de interés}

Los autores no declaran conflicto de interés.

\section{Financiación}

El presente trabajo no tuvo financiación.

\section{Agradecimientos}

A la Escuela de Odontoogía de la Universidad del Valle.

\section{Referencias}

1. Corbera E. Fundamentación teórica de la Bioneuroemoción. La evolución de la Biodescodificación. Primera ed. EDITORIAL SJ, editor. Torreon- Mexico: Universidad Iberoamericana Torreon; 2015. $43 \mathrm{p}$.

2. Casals MML. Desprogramación Biológica y Disposiciones corporales: Una herramienta a través del Coaching para la Salud [Internet].

3. Mirre JC. Biodescodificación: un nuevo paradigma de salud. Discovery Salud [Internet]. 2015 Jan;2.

4. Molina-jiménez T, Gutiérrez-garcía A, Hernández-Domínguez L, Contreras CM. Estrés psicosocial : Algunos aspectos clínicos y experimentales. An Psicol. 2008;24(2):353-60.

5. Duval F, Félix González HR. Neurobiología del estrés. REV CHIL NEURO-PSIQUIAT. 2010;48(4):307-18.

6. Baum A. Stress , Intrusive Imagery , and Chronic Distress. Heal Psychol. 1990;(6):653-75.
7. Bernabé DG, Tamae AC, Biasoli ÉR, Oliveira SHP. Brain, Behavior, and Immunity Stress hormones increase cell proliferation and regulates interleukin-6 secretion in human oral squamous cell carcinoma cells. Brain, Behav Inmunity. 2011;25:574-83.

8. Bowen Zhang, Chenzhou Wu, Wen Chen, Ling Qiu, Shensui Li, Tao Wang, Huixu Xie, Yi Li, Chunjie Li LL. The stress hormone norepinephrine promotes tumor progression through $\beta 2$ adrenoreceptors in oral cancer. Arch Oral Biol. 2020;113:10.

9. Lostaunau V, Torrejón C. Estrés, afrontamiento y calidad de vida relacionada a la salud en mujeres con cáncer de mama. Actual en Psicol. 2017;31(122):75-90.

10. Zhang Y, Zanos P, Jackson IL, Zhang X, Zhu X, Gould T, et al. Psychological stress enhances tumor growth and diminishes radiation response in preclinical model of lung cancer. Radiother Oncol [Internet]. 2020;146:126-35. Available from: https://doi.org/10.1016/j.radonc.2020.02.004

11. Pandey AK, Gupta A, Tiwari M, Prasad S, Pandey AN, Yadav PK, et al. Impact of stress on female reproductive health disorders: Possible bene fi cial e ff ects of shatavari ( Asparagus racemosus ). Biomed Pharmacother. 2018;103(January):46-9.

12. Uribe Londoño F, Gómez Franco J, Mesa Franco LF, Lezcano Tobón LA. Ejes neuroendocrinos del estrés, síndrome metabólico y alteraciones psiquiátricas del síndrome de Cushing. Iatreia. 2005; 18(4):431-45

13. Aleksejunñiené J, Holst D, Eriksen HM, Gjermo P. Psychosocial stress, lifestyle and periodontal health. J Clin Periodontol. 2002;29(4):326-35.

14. Oh SH, Bae BG, Park CO, Noh JY, Park IH, Wu WH, et al. Association of stress with symptoms of atopic dermatitis. Acta Derm Venereol. 2010;90(6):582-8.

15. Flint MS, Bovbjerg DH. DNA damage as a result of psychological stress : implications for breast cancer. Breast Cancer Res. 2012;14(5):3-5.

16. Kelly RR, Mcdonald LT, Jensen NR, Sidles SJ, Martino G. Impacts of Psychological Stress on Osteoporosis : Clinical Implications and Treatment Interactions. Front Psychiatry. 2019;10(April):1-21.

17. Donahue CP, Kosik KS, Shors TJ. Growth hormone is produced within the hippocampus where it responds to age , sex , and stress. PNAS. 2006;103(15).

18. Azuma K, Adachi Y, Hayashi H, Kubo KY. Chronic psychological stress as a risk factor of osteoporosis. J UOEH. 2015;37(4):245-53.

19. Briot K, Roux C. Glucocorticoid-induced osteoporosis. 2015;

20. García-llana H, Remor E, Peso G, Selgas R. El papel de la depresión, la ansiedad, el estrés y la adhesión al tratamiento en la calidad de vida relacionada con la salud en pacientes en diálisis : revisión sistemática de la literatura. Nefrología. 2014;34(5).

21. Davidopoulou S, Chatzigianni A. Craniofacial morphology and dental maturity in children with reduced somatic growth of different aetiology and the effect of growth hormone treatment. Prog Orthod. 2017;4-11.

22. Miller GE, Murphy MLM, Cashman R, Ma R, Ma J, Arevalo JMG, et al. Greater inflammatory activity and blunted glucocorticoid signaling in monocytes of chronically stressed caregivers. BRAIN, Behav Immun [Internet]. 2014;(June). Available from: http://dx.doi.org/10.1016/j.bbi.2014.05.016

23. Rongen-Westerlaken C, Born E vd, Prahl-Andersen B, Teunenbroek A v., Manesse P, Otten B, et al. Effect of growth hormone treatment on craniofacial growth in Turner's syndrome. Acta Pædiatrica. 1993;82(4):364-8.

24. Jones A, Godfrey KM, Wood P, Osmond C, Goulden P, Phillips DIW. Fetal Growth and the Adrenocortical Response to Psychological Stress. JClin Endocrinol Metab. 2006;91(5):1868-71.

25. Lostaunau V, Torrejón C, Cassaretto M. Estrés, afrontamiento y calidad de vida relacionada a la salud en mujeres con cáncer de mama. Actual en Psicol. 2017;31(122):75.

26. Chatterjee S, Mondal S. Effect of Regular Yogic Training on Growth Hormone and Dehydroepiandrosterone Sulfate as an Endocrine Marker of Aging. Hindawi. 2014;2014:16.

27. Domené HM, Fierro-Carrión G. Genetic disorders of GH action pathway. Growth Horm IGF Res. 2018;38(December):19-23.

28. Litsas G. Growth Hormone and Craniofacial Tissues. An update. Open Dent J. 2015;9(1):1-8. 
29. Xie H, Li C, He Y, Griffin R, Ye Q, Li L. Chronic stress promotes oral cancer growth and angiogenesis with increased circulating catecholamine and glucocorticoid levels in a mouse model. ORAL Oncol [Internet]. 2015; Available from: http://dx.doi.org/10.1016/j.oraloncology.2015.08.007

30. Preda SA, Albulescu DM, Mitroi MR, Popescu M, Nechita F, Camen A, et al. Craniofacial morphology aspects in children with isolated growth hormone deficiency - A cephalometric study. Rom J Morphol Embryol. 2019;60(2):653-8.

31. Pirinen S. Endocrine regulation of craniofacial growth. 1995;(15):179-85.

32. Wójcik D, Beń-Skowronek I. Craniofacial morphology in children with growth hormone deficiency and Turner syndrome. Diagnostics. 2020;10(2).

33. Juloski J, Dumančić J, Šćepan I, Lauc T, Milašin J, Kaić Z, et al. Growth hormone positive effects on craniofacial complex in Turner syndrome. Arch Oral Biol. 2016;71:10-5.

34. Funatsu M, Sato K, Mitani H. Effects of growth hormone on craniofacial growth: Duration of replacement therapy. Angle Orthod. 2006;76(6):970-7.

35. Kjellberg H, Beiring M, Wikland KA. Craniofacial morphology, dental occlusion, tooth eruption, and dental maturity in boys of short stature with or without growth hormone deficiency. Eur J Oral Sci. 2000;108(5):359-67.

36. Domínguez-Menéndez G, Cifuentes L, González C, Lagos M, Quiroga T, Rumié H, et al. Growth hormone of dried blood spot for the diagnosis of growth hormone deficiency. Rev Chil Pediatr. 2019;90(2):145-51.

37. Silva N, Bullinger M, Sommer R, Rohenkohl A, Witt S, Quitmann J. Children's psychosocial functioning and parents' quality of life in paediatric short stature: The mediating role of caregiving stress. Clin Psychol Psychother. 2018;25(1):e107-18.

38. Batty GD, Shipley MJ, Gunnell D, Huxley R, Kivimaki M, Woodward M, et al. Economics and Human Biology Height , wealth, and health: An overview with new data from three longitudinal studies. Econ Hum Biol. 2009;7:137-52.

39. Erling A, Wiklund I, Wikland KA. Psychological functioning in boys of short stature: effects of different levels of growth hormone secretion. Acta Paediatr. 2002;91(15):966-71.

40. Sandberg DE, Gardner M. Short Stature: Is It a Psychosocial Problem and Does Changing Height Matter? Pediatr Clin North Am. 2015;62(4):963-82.

41. Harada D, Seino Y. Growth hormone and bone. Bone Drugs Pediatr Effic Challenges. 2014;19(April):117-34.

42. Forsberg C, Krekmanova L, Dahllöf G. The effect of growth hormone therapy on mandibular and cranial base development in children treated with total body irradiation. Eur J Orthod. 2002;24:285-92.

43. Van De Kelft AS, Lievens C, De Groote K, Demulier L, De Backer J, T'Sjoen G, et al. Disproportion and dysmorphism in an adult Belgian population with Turner syndrome: risk factors for chronic diseases? Acta Clin Belgica Int J Clin Lab Med. 2020;75(4):258-66.

44. Choi SH, Fan D, Hwang MS, Lee HK, Hwang CJ. Effect of growth hormone treatment on craniofacial growth in children: Idiopathic short stature versus growth hormone deficiency. J Formos Med Assoc. 2017;116(4):313-21.

45. Simmons KE. Growth Hormone and Craniofacial Changues: Preliminary data From Studies in Turner' s Syndrome. Am Acad Pediatr. 1999;104-1021.

46. Juloski J, Glisic B, Scepan I, Milasin J, Mitrovic K, Babic M. Ontogenetic changes of craniofacial complex in Turner syndrome patients treated with growth hormone. Clin Oral Investig. 2013;17(6):1563-71

47. Di F, Cazzolla A, Lacarbonara V, Testa N, Zhurakivska K, Lo Muzio L E al. Tratamiento ortopédico ortodóntico del paciente con sindrome de turner: revisión de la literatura y presentación de un caso. Rev Latinoam Ortod y Odontopediatria. 2018;38:239-48.

48. Zenker S, Haverkamp F, Klingmüller D. Growth hormone deficiency in pituitary disease: Relationship to depression, apathy and somatic complaints. Eur J Endocrinol. 2002;147(2):165-71. 